\title{
Intento e ideación suicida y su asociación con el abuso sexual en adolescentes escolarizados de Boyacá - Colombia*
}

\section{Suicidal ideation and attempt and their relation to sexual abuse in students of Boyacá - Colombia}

\author{
Mónica Patricia Pérez Prada \\ ORCID: 0000-0002-6204-3175 \\ Lizeth Cristina Martínez Baquero \\ ORCID: 0000-0002-3673-1492 \\ Mildred Alexandra Vianchá Pinzón \\ ORCID: 0000-0001-9438-8955 \\ Universidad de Boyacá, Colombia \\ Bertha Lucía Avendaño Prieto** \\ ORCID: 0000-0002-3006-7981 \\ Universidad Católica de Colombia \\ Recibido: 21 de septiembre de 2016 \\ Revisado: 13 de octubre de 2016 \\ Aceptado: 3 de noviembre de 2016
}

\section{Resumen}

El objetivo de este estudio empírico fue identificar la presencia del intento e ideación suicida, abuso sexual y la asociación entre estas variables en estudiantes de educación secundaria de tres ciudades del departamento de Boyacá. Se contó con 1292 participantes cuyas edades oscilaron entre 12 y 20 años. Los instrumentos utilizados fueron una Encuesta Sociodemográfica y ellnventario de Ideación Suicida Positiva y Negativa. Se hallaron diferencias estadísticamente significativas en las variables estudiadas. Se identifica que los jóvenes que han sido víctimas de abuso sexual por penetración, han tenido intento suicida (1.9\%); además, el $48 \%$ de las victimas presentan ideación suicida.

Palabras Clave: intento suicida, ideación suicida, abuso sexual, adolescente.

Artículo de investigación. El artículo es resultado del proyecto titulado "Variables psicológicas, familiares y sociodemográficas relacionadas con la ideación y acto suicida en adolescentes escolarizados de Tunja, Sogamoso y Duitama"; financiado por la fundación para la promoción de la investigación y la tecnología. Proyecto numero: 3.190. (doi: http://dx.doi.org/10.15332/s1794-9998.2017.0001.08)

... Bertha Lucia Avendaño, Universidad Católica de Colombia, correo electrónico: blavendano@ucatolica.edu.co. Mónica Patricia Pérez Prada, correo electrónico: mpperez@uniboyaca.edu.co, dirección postal: carrera 2.a este \# 4-169, Tunja, Boyacá, Colombia. 


\section{Abstract}

The purpose of this descriptive study was to identify the presence of suicidal attempt and suicidal ideation, sexual abuse and the relationship among these variables, in high school students from three towns in Boyaca, Colombia. This study was conducted with 1,292 participants whose ages ranged between 12 and 20 years old. The instruments used were a sociodemographic survey and the Positive and Negative Suicide Ideation Inventory. Statistically significant differences in the variables studied were found. Among the most relevant findings of this research, it was found that young victims of sexual abuse with penetration have had suicidal attempts (1.9\%); moreover, $48 \%$ of the victims have suicidal ideation.

Keywords: suicidal attempt, suicidal ideation, suicidal behavior, sexual abuse, adolescent.

\section{Introducción}

La muerte es uno de los fenómenos individuales que mayor impacto genera en el núcleo social de arraigo, la certeza de la ausencia y la radicalidad de esta han movilizado diferentes acciones de la humanidad para hacer frente a dicha situación. Una de sus manifestaciones es el suicidio, definido como el acto autoinfligido con resultado fatal, anticipado y llevado a cabo por la propia persona, con conocimiento y propósito de obtener un cambio deseado (Organización Mundial de la Salud OMS, 2012), es considerado la segunda causa de muerte en personas de 15 a 29 años (OMS, 2014) y un problema de salud pública internacional (OMS, 2001), de hecho se estima que más de 800000 personas se suicidan cada año y el $75 \%$ de ellos se producen en países de ingresos bajos y medios (OMS, 2014).

La conducta suicida de acuerdo con Toro, Avendaño y Castrillón (2016) es definida como toda acción producida con el propósito explícito o implícito de quitarse la vida, que incluye cualquier intención, ideación o pensamiento que puede finalizar en la muerte. Sin embargo, en el estudio del comportamiento suicida se proponen principalmente tres conceptos: ideación suicida, intento suicida y suicidio. La ideación suicida se considera como pensamientos o ideas de servirse como agente de la propia muerte, que expresa el deseo o la intención de morir, y puede variar en gravedad depen- diendo de la especificidad del plan suicida y del grado de intención (Toro, Avendaño y Castrillón, 2016), sumado a lo anterior, Mingote, Jiménez, Osorio y Palomo (2004) proponen que estas manifestaciones deben mantenerse por lo menos durante dos semanas. En la literatura se identifican distintos trabajos científicos que han enfocado el estudio de la ideación como un factor predictor o factor de riesgo del suicidio (Cabra, Infante y Sossa, 2010; Córdova, Cubillas y Román, 2011; Pérez, Ibáñez, Reyes, Atuesta y Suárez, 2008), no obstante, el suicidio puede comenzar o no con la idea de perder la vida, es decir, no todos los suicidios consumados han sido antecedidos por la ideación suicida, ya que se pueden presentar de manera impulsiva (González, Arana y Jiménez, 2008).

Por otro lado, el intento suicida es entendido como toda acción autoinfligida con el propósito de hacerse daño de manera letal y cuyo resultado no fue la muerte, muchas veces es considerado como conducta suicida no fatal por la forma de presentarse (OMS, 2012).

De acuerdo con los reportes estadísticos de Medicina Legal y Ciencias Forenses (2014), en Colombia en el 2013 el suicidio fue la cuarta causa de muerte violenta en el país, con una tasa de 3.84 por cada 100000 habitantes (1810 casos). Además, la OMS (2012) afirma que Colombia es el tercer país con mayor número de muertes por suicidio después de Cuba y Brasil. Es importante exponer que Boyacá 
en el 2010 se ubicó como el segundo departamento del país con mayor cantidad de suicidios, siendo Tunja y Sogamoso las ciudades más afectadas (Instituto Nacional de Medicina Legal, 2010).

Ante el panorama mundial y nacional, diferentes investigaciones se han propuesto explicar la etiología de este fenómeno y concluyen que es el resultado de la interacción de factores biológicos, genéticos, sociales, culturales y ambientales (Pérez, Viacha, Martínez y Salas, 2013; Caycedo et al., 2010), entre los cuales se encuentra el abuso sexual y el maltrato familiar como factores de riesgo. Cortés, Cantón-Cortés y Cantor-Duarte (2011) encontraron que la ideación e intento suicida es una de las consecuencias a largo plazo del abuso sexual. Redundando con lo anterior, Eisenberg (2007) encontró que los estudiantes de bachillerato que habían sido víctimas de abuso sexual infantil presentaban mayor riesgo de conducta suicida. Conviene señalar que Cabra, Infante y Sossa (2010), realizaron una revisión de la literatura existente en diferentes bases de datos sobre los factores de riesgo para la conducta suicida y encontraron que el abuso sexual se relaciona con el suicidio en adolescentes.

En este estudio se entiende el abuso sexual como todo acceso/acto/estimulación sexual que una persona con suficiente experiencia ejerce contra un niño, niña o adolescente que se encuentra en desventaja frente a su agresor (Rodríguez, 2011). En Colombia el Instituto Nacional de Medicina Legal y Ciencias Forenses registró 21115 exámenes médico legales por presunto delito sexual en Colombia, lo que representa un aumento de $376 \mathrm{ca}-$ sos en relación con el 2013; asimismo se expone que el 85.09 \% de los casos reportados corresponden a mujeres y el rango de edad más afectado es el comprendido entre los 10 y 14 años.

De lo expuesto con anterioridad se infiere que tanto la conducta suicida como el abuso sexual son problemáticas relacionadas que afectan la salud mental y física de las víctimas, de sus núcleos sociales y familiares, razón que justifica su estudio para generar conocimientos que orienten acciones encaminadas a mitigar los efectos negativos de estos hechos sobre los seres humanos y las comunidades.

\section{Método}

\section{Tipo de investigación}

El estudio se realizó con metodología cuantitativa, presenta datos empíricos originales producidos por las autoras, descriptivo de poblaciones mediante cuestionario y autoreporte (Ato, López y Benavente, 2013).

\section{Población}

85444 jóvenes de los grados octavo, noveno, décimo y undécimo de instituciones educativas públicas de las tres ciudades con mayor número de habitantes del departamento de Boyacá (Colombia), Tunja, Sogamoso y Duitama (Ministerio de Educación Nacional, 2013).

\section{Muestra}

Los participantes de la investigación se seleccionaron mediante muestreo probabilístico estratificado, las variables de estratificación fueron ciudad, institución educativa y grado escolar (Hernández, Fernández y Baptista, 2010). Con un error de muestreo de 0,022, el tamaño muestral indicado era de 1500 estudiantes; sin embargo, la muestra estuvo conformada por 1292 estudiantes, ya que se excluyeron los cuestionarios en donde se identificaron datos perdidos. Como criterio de inclusión se tuvo en cuenta la voluntad de participar en el estudio y la firma del consentimiento informado, se excluyeron estudiantes con discapacidad cognitiva y los jóvenes que no pertenecían a instituciones educativas públicas de las 3 ciudades mencionadas.

La fórmula 1 (Daniel, 2008) muestra los indicadores utilizados.

$$
\mathrm{n}=\frac{N p a Z^{2}}{(N-1)^{2} e^{2}+Z^{2} p q} \quad \begin{aligned}
& \mathrm{N}=85444 \\
& \mathrm{p}=0,13 \\
& \mathrm{q}=0,87 \\
& \mathrm{Z}=2576 \\
& \mathrm{e}=0,022
\end{aligned}
$$

Teniendo en cuenta el número de estudiantes de cada ciudad, el tamaño de la muestra se distribu- 
yó de la siguiente forma, Tunja: 668 estudiantes, Sogamoso: 439, Duitama: 493.

\section{Instrumentos}

Inventario de Ideación Suicida Positiva y Negativa (Positive and Negative Suicidal Ideation - PANSI), instrumento adaptado por medio de la técnica de traducción simple con población colombiana por Villalobos (2010). El PANSI elaborado por Osman, Gutiérrez, Kopper, Barrios y Chiros (1998) es un cuestionario de 14 ítems, 6 de ideación suicida positiva (factores protectores) y 8 de ideación suicida negativa (riesgo suicida), evaluados en el marco de las últimas dos semanas y en los que se pregunta qué tan a menudo la persona ha presentado cada uno de los 14 pensamientos. El encuestado debe responder haciendo uso de una escala de 5 puntos que oscila entre 0 (nunca) y 4 (siempre). Este inventario ha mostrado coeficientes de consistencia interna superiores a 0,8 y adecuada validez de constructo (concurrente y discriminante), tanto en muestras clínicas como no clínicas (Osman et al., 2003) y consta de 2 escalas factorialmente derivadas: ideación positiva e ideación negativa.

Autoreporte de abuso sexual e intento suicida, este instrumento fue elaborado por las autoras y sometido a juicio de expertos, consta de 4 ítems cerrados que indagan sobre el intento suicida y la presencia de abuso sexual en alguna etapa de la vida.

\section{Definición de variables clínicas}

Ideación suicida: pensamientos o ideas de servirse como agente de la propia muerte, que expresa el deseo o la intención de morir, que puede variar en gravedad dependiendo de la especificidad del plan suicida y del grado de intención (Toro, Avendaño y Castrillón, 2016).

Intento suicida: acción autoinfligida con el propósito de hacerse daño de manera letal y cuyo resultado no fue la muerte (OMS, 2012).

Abuso sexual: todo acceso/acto/estimulación sexual en el cual una persona con suficiente expe- riencia ejerce contra un niño, niña o adolescente que se encuentra en desventaja frente a su agresor (Rodríguez, 2011).

\section{Procedimiento}

Se contactó a los secretarios de educación, rectores y coordinadores de las 3 ciudades para solicitar la autorización. Se seleccionaron únicamente las instituciones de carácter público. Posteriormente se escogieron al azar estudiantes que cursaran los grados octavo a undécimo, seleccionando por institución el número de estudiantes establecido en el muestreo.

A continuación se enviaron los consentimientos informados a los padres de familia para ser diligenciados y entregados el día de la aplicación del instrumento. Se les explicó a los estudiantes el objetivo del estudio y la forma en que se usaría la información suministrada, también se les solicitó dar su asentimiento para participar en la actividad. Se explicó el diligenciamiento del instrumento siguiendo los patrones consignados en la prueba.

Teniendo en cuenta el nivel de medición de las variables, el análisis de resultados se realizó con el software SPSS versión 2.0.

La presente investigación se ajustó a la legislación colombiana vigente de la resolución número 8430 de 1993 (Ministerio de Salud Nacional, 1993) y de la ley 1090 de 2006 (Ministerio de Protección Social y Ministerio de Educación, 2006), en las cuales se dictan las normas para la investigación en salud y la práctica de investigaciones en psicología. Asimismo, fue avalada por el Comité de Bioética institucional.

\section{Resultados}

Inicialmente se mostrarán las características sociodemográficas de los jóvenes en quienes se identificó la presencia de intento e ideación suici$\mathrm{da}$, seguida de abuso sexual y finalmente se presentarán las asociaciones que resultaron estadísticamente significativas. 


\section{Intento suicida}

Respecto al intento suicida y al sexo los resultados de este estudio señalan que el $15.5 \%$ de los participantes (198 estudiantes) han intentado suicidarse, de este total el $60.1 \%$ (119 estudiantes) corresponden al sexo femenino y el $39.9 \%$ al sexo masculino. El grupo de edad con mayor número de intentos de suicidio oscila entre los 14 y 16 años, con un porcentaje de $73.22 \%$.

En relación con el tipo de familia, se halló que en la compuesta se encuentra el mayor número de adolescentes con intento suicida, en términos porcentuales corresponde al $21 \%$; seguido de la familia monoparental con $17 \%$; la nuclear con un $14 \%$ y la extensa con el $13 \%$, no obstante, hay que mencionar que el porcentaje mayor se encuentra en la opción no marco con un $33.3 \%$, el mayor porcentaje de estudiantes que no ha intentado suicidarse corresponde a la familia nuclear (63\%), seguido de monoparental (21\%), extensa ( $8 \%)$, compuesta (5 $\%)$ y el porcentaje restante no marco (3\%).

\section{Ideación suicida}

Según los resultados del PANSI el 17.6 \% (226 personas) presentan ideación suicida negativa, con mayor frecuencia en el género femenino (tabla 1). De igual manera el grupo etáreo más afectado es el comprendido entre los 14 y 17 años.

Tabla 1.

Contingencia entre ideación suicida y sexo.

\begin{tabular}{|c|c|c|c|}
\hline \multirow{2}{*}{ Ideación suicida } & \multicolumn{2}{|c|}{ SEXO } & \multirow{2}{*}{ TOTAL } \\
\hline & Femenino & Masculino & \\
\hline \multirow{2}{*}{ Con riesgo } & 135 & 91 & 226 \\
\hline & $10.5 \%$ & $7.1 \%$ & $17.6 \%$ \\
\hline \multirow{2}{*}{ Sin riesgo } & 568 & 485 & 1053 \\
\hline & $44.5 \%$ & $37.9 \%$ & $82.4 \%$ \\
\hline Total & 703 & 576 & 1279 \\
\hline
\end{tabular}

Fuente: elaboración propia.

Respecto al tipo de familia, se encontró que el mayor porcentaje de estudiantes con riesgo de ideación suicida ( $36.1 \%$ ) pertenecen a la familia compuesta, en las demás, los porcentajes son inferiores al $18 \%$.

\section{Abuso sexual}

Para este estudio el abuso sexual se clasificó en: abuso sexual por tocamientos y abuso sexual por penetración. Con el fin de determinar el abuso sexual por tocamientos en la población participante, se incluyó en el cuestionario sociodemográfico la pregunta: "ha sido víctima de abuso sexual: tocamientos, manoseos, caricias en los genitales, o alguna parte del cuerpo, que lo haga sentir incomodo, sin su consentimiento", entre los hallazgos se encuentra un total de 70 reportes de abuso sexual por tocamientos que equivale al $5.4 \%$ del total de la muestra, del cual el $71.4 \%$ corresponde a mujeres y el $28.6 \%$ a hombres.

En relación con el abuso sexual por penetración, el $1.9 \%$ (25 jóvenes) reportan haber sido víctimas de este flagelo, se identifican 8 casos de hombres y 17 de mujeres. Se encontró que el mayor número de casos, tanto de abuso sexual por penetración como por tocamientos, está en la edad de 16 años. En cuanto al tipo de familia, la nuclear es en la que más se identificaron casos, esta situación puede ser explicada porque el mayor número de participantes de la muestra pertenecían a esta clasificación.

A continuación se exponen los resultados de las asociaciones entre variables que resultaron estadísticamente significativas.

\section{Ideación suicida y abuso sexual}

Las tablas de contingencia y las pruebas chi-cuadrado para la ideación suicida se realizaron teniendo en cuenta las dos formas de abuso sexual expuestas anteriormente.

\section{Abuso sexual por tocamientos}

Para considerar la asociación entre abuso sexual por tocamientos e ideación suicida, se realizó una prueba chi-cuadrado de Pearson cuyos resultados mostraron un valor de chi de 18 638, y una sig bilateral menor de 0.05 asociación significativa entre estas dos variables. La tabla 2 indica que del total de estudiantes que presenta riesgo de ideación 
suicida, el $11.5 \%$ ha sido víctima de abuso sexual por tocamientos, mientras que este porcentaje en el grupo de estudiantes sin riesgo de ideación suicida, corresponde al $4.2 \%$.

Tabla 2.

Contingencias entre abuso sexual por tocamientos con ideación suicida.

\begin{tabular}{|c|c|c|c|c|}
\hline \multicolumn{4}{|c|}{ Abuso sexual tocamientos PANSI Negativo } & \multirow[t]{2}{*}{ Total } \\
\hline & Sin riesgo & & Con riesgo & \\
\hline \multirow{2}{*}{ sí } & 45 & & 26 & 71 \\
\hline & $4.2 \%$ & & $11.5 \%$ & \\
\hline \multirow{2}{*}{ NO } & 1015 & & 201 & 1216 \\
\hline & $95.8 \%$ & & $88.5 \%$ & \\
\hline TOTAL & 1060 & $100 \%$ & $100 \%$ & 1287 \\
\hline
\end{tabular}

Fuente: elaboración propia.

\section{Abuso sexual por penetración}

La tabla 3 señala que el $52 \%$ de quienes han sufrido este tipo de abuso sexual presentan riesgo de ideación suicida. El $17 \%$ de quienes no han sufrido abuso sexual por penetración están en riesgo de ideación suicida.

Tabla 3.

Contingencia entre abuso sexual por penetración e ideación suicida.

\begin{tabular}{|c|c|c|c|}
\hline \multirow{2}{*}{$\begin{array}{l}\text { Abuso sexual } \\
\text { penetración }\end{array}$} & \multicolumn{2}{|c|}{ PANSI Negativo } & \multirow{2}{*}{ Total } \\
\hline & Sin riesgo & Con riesgo & \\
\hline \multirow{2}{*}{ sí } & 12 & 13 & 25 \\
\hline & $48,0 \%$ & $52,0 \%$ & $100,0 \%$ \\
\hline \multirow{2}{*}{ NO } & 1047 & 214 & 1261 \\
\hline & $83,0 \%$ & $17,0 \%$ & $100,0 \%$ \\
\hline \multirow{2}{*}{ Total } & 1059 & 227 & 1286 \\
\hline & $82,3 \%$ & $17,7 \%$ & $100,0 \%$ \\
\hline
\end{tabular}

Fuente: elaboración propia.

El valor arrojado por la prueba chi-cuadrado para determinar la relación entre abuso sexual por penetración e ideación suicida es de 20694 y una significancia bilateral menor de 0.05 , lo que permite concluir que existe una asociación significativa entre las dos variables.

\section{Intento suicida y abuso sexual}

A continuación se presentan las contingencias y la pruebas chi-cuadrado para el intento suicida y el abuso sexual.

\section{Abuso sexual por tocamientos}

De los 70 estudiantes que han sido víctimas de abuso sexual por tocamientos, el $68.5 \%$ (48) han intentado suicidarse, este porcentaje en los estudiantes que no han sido víctimas de abuso sexual por tocamientos corresponde al $12.5 \%$.

Los valores obtenidos en la prueba chi-cuadrado para las variables intento suicida y abuso sexual por tocamientos fue de 159068 y una significancia de 0.00 .

\section{Abuso sexual por penetración}

La tabla 4 muestra las contingencias entre abuso sexual por penetración y el intento suicida. Se observa que todos los estudiantes que han sido víctimas de este tipo de abuso sexual (25) han intentado suicidarse.

Tabla 4.

Contingencias entre abuso sexual por penetración e intento suicida.

\begin{tabular}{llcl}
\hline & \multicolumn{2}{c}{$\begin{array}{c}\text { Abuso sexual } \\
\text { penetración }\end{array}$} & Total \\
\cline { 2 - 3 } & Sí & No & \\
\hline $\begin{array}{l}\text { Sí ha intentado } \\
\text { suicidarse }\end{array}$ & 25 & 175 & 200 \\
\hline $\begin{array}{l}\text { No ha intentado } \\
\text { suicidarse }\end{array}$ & 0 & 1083 & 1083 \\
\hline Total & 25 & 1258 & 1283 \\
\hline
\end{tabular}

Fuente: elaboración propia.

El valor del chi-cuadrado fue 138065 y la significancia de 0.00 .

\section{Discusión}

El intento suicida se ha constituido en el predictor más potente para el suicidio consumado, una 
persona que lo ha intentado tiene mayor probabilidad de volver a intentarlo (Cabra, Infante y Sossa, 2010), es un fenómeno común en la población adolescente y su incidencia aumenta en la adolescencia media (Rueda, Rangel, Castro y Camacho, 2010; Ventura et al., 2010). En el presente estudio, se encontró que el $15.5 \%$ de los participantes (198 estudiantes) han intentado suicidarse y sus edades oscilan entre los 14 y los 17 años; a partir de la información expuesta anteriormente, con los reportes de la Organización Mundial de la Salud y del Instituto Nacional de Medicina Legal y Ciencias Forenses, es posible afirmar que las personas que han intentado suicidarse están en alto riesgo de suicidio.

Algo semejante ocurre con el sexo, esta variable en la literatura se ha identificado como una de las mejores predictores del intento suicida; para el Instituto Nacional de Salud (2016) y el Instituto Nacional de Medina Legal y Ciencias Forenses (Forensis, 2014) en Colombia las mujeres intentan suicidarse con mayor frecuencia, pero los hombres son quienes mayor número de suicidios consumados reportan; información que coincide con lo encontrado en el presente estudio, ya que el $60.1 \%$ de los estudiantes que han intentado suicidarse corresponden al sexo femenino. En este sentido, Rodríguez, Medina y Cardona (2013); Rueda et al. (2010) y Cabra et al. (2010); afirman que los intentos de suicidio son más frecuentes en mujeres adolescentes que en adultas, y que es mayor el número de mujeres adolescentes que consultan por suicidabilidad.

En relación con el tipo de familia se halló que el mayor porcentaje de los estudiantes con intento suicida pertenecen a la compuesta, resultados que corroboran los hallazgos presentados por Carmona et al. (2010) y Cañón (2011) quienes afirman que los miembros de familias separadas presentan mayor riesgo de conducta suicida, al mismo tiempo identifican que los problemas y el grado de disfunción familiar, son el principal precipitante de la conducta suicida. Así mismo, Bella, Fernández y Willington (2010) aseveran que las relaciones de alto riesgo con el padre o la madre hacen parte de los factores familiares de riesgo frente a la ideación suicida, especialmente en niños y jóvenes.
Con respecto a lo anterior, la ideación suicida ha sido una variable ampliamente estudiada como predictor y factor de riesgo ante la conducta suicida, se determinó que 226 personas (17.6\%) de la población estudiada presentan esta condición; información que es coherente con la literatura científica a nivel internacional, la cual registra que entre el $11 \%$ y $49 \%$ de la población escolar presenta ideación suicida (Centers for Disease Control, citado por Carvajal y Caro, 2011). Asimismo, el estudio comprobó mayor frecuencia en las mujeres como lo muestran las investigaciones realizadas por Amézquita, González y Zuluaga (2008); y Siabato y Salamanca (2015).

Preocupa que la conducta suicida sea más frecuente en grupos de edad cada vez menores, estos resultados ponen de manifiesto la situación, ya que el grupo en el cual se encontró mayor frecuencia de ideación suicida (64.1\%) fue el comprendido entre los quince y los diecisiete años, resultados confirmados en estudios similares; entre ellos el realizado por Amézquita, González y Zuluaga (2008) quienes identificaron este mismo rango de edad en una población de adolescentes escolarizados en Manizales. En Chile, Ventura et al. (2010) encontraron que la edad en la cual se presentó el mayor porcentaje de ideación suicida fue quince años. No obstante, en el estudio realizado por Paniagua, González y Rueda (2014) en Medellín encontraron que la edad comprendida entre los once y catorce años están en riesgo de conducta suicida, evidenciando que la población en riesgo es cada vez menor.

De manera similar a lo que ocurre con el intento suicida, es en la familia compuesta donde se presenta mayor número de personas con ideación, esta información coincide con lo encontrado por Córdova, Rosales y García (2012) quienes afirman que existe asociación entre ideación suicida y el bajo apoyo familiar, asimismo las familias desligadas en las cuales los padres muestran actitud fría, no comunicativa e intolerante hacia sus hijos representan un factor de riesgo (Muñoz et al., 2005).

Prosiguiendo con el análisis, el abuso sexual en niños, adolescentes y adultos es más común de lo que se piensa, estudios han evidenciado que 1 de 
cada 4 niñas y 1 de cada 8 niños serán sexualmente agredidos antes de cumplir los dieciséis años (Contreras, Bott, Guedes y Dartnall, 2010). En Colombia en el caso particular de la violencia sexual, el $86 \%$ de los exámenes médico legales se realizan a menores de 18 años, la población más afectada son las niñas entre los 10 y los 14 años (Instituto de Bienestar Familiar, 2012), dicha información es confirmada por los resultados de este estudio, en el cual se identifica mayor número de víctimas de abuso sexual por penetración y tocamientos en la población femenina comprendida entre los $14 \mathrm{y}$ 17 años.

Uno de los hallazgos más preocupantes de esta investigación es que todos los estudiantes víctimas de abuso sexual por penetración, que participaron en esta investigación, han tenido intento suicida; además, el $48 \%$ de las victimas presentan ideación suicida, lo cual invita a considerar la asociación entre abuso sexual y suicidio para emprender acciones preventivas que involucren estas dos variables. Casullo (2004) reveló que entre el $6 \%$ y $13 \%$ de los jóvenes, informaron que intentaron quitarse la vida por lo menos una vez, siendo todos ellos víctimas de violaciones sexuales. Se hace evidente que siguen siendo las mujeres las más vulnerables a este fenómeno, pero resulta preocupante que el porcentaje de personas que reportaron abuso sexual en esta investigación sea superior a los datos que presentan en otras investigaciones, como la de Chávez et al. (2009).

Para añadir, los efectos psicológicos y sociales que el abuso sexual produce en quienes han padecido esta experiencia en cualquier etapa de la vida, provee consecuencias a corto, mediano y largo plazo. Por lo que las secuelas psicológicas que se han relacionado con la experiencia de abuso sexual infantil pueden perdurar a lo largo del ciclo evolutivo y configurar, en la edad adulta, el desarrollo de problemas emocionales y afectivos como ansiedad, depresión, consumo abusivo de alcohol y drogas, promiscuidad sexual e incluso intentos e ideación suicida (Rodríguez, 2011).

Respecto a la asociación encontrada entre intento suicida y abuso sexual, Villalobos (2010) afirma que los eventos estresantes con el abuso sexual se han constituido en un factor de riesgo para el suicidio, de esta investigación se concluye que el abuso sexual por penetración y tocamientos está relacionado con la conducta suicida, tanto con el intento como con la ideación; situación que debe ser tenida en cuenta para diseñar programas y políticas que prevengan y mitiguen la problemática del suicidio; de las misma manera los resultados de este estudio deben encender las alarmas de las autoridades y las instituciones frente al fenómeno del abuso sexual, ya que como se mencionó anteriormente el porcentaje hallado de víctimas de este flagelo es superior al de otras poblaciones.

\section{Referencias}

Amézquita, M., González, R., y Zuluaga, D. (2008). Prevalencia de la depresión e ideación suicida en estudiantes de 8, 9, 10 y 11 grado, en ocho colegios oficiales de Manizales. Hacia la Promoción de la Salud, 13, 143-153. Recuperado de http://www.scielo.org.co/pdf/hpsal/ v13n1/v13n1a09.pdf

Ato, M., López, J., y Benavente, A. (2013). Un sistema de clasificación de los diseños de investigación en psicología. Anales de Psicología, 29(3), 1038-1059. Recuperado de http://www. redalyc.org/articulo.oa?id=16728244043

Bella, M., Fernández, R., y Willinton, J. (2010). Intento de suicidio en niños y adolescentes: depresión y trastorno de conducta disocial como patologías más frecuentes. Archivos argentinos de pediatría, 108(2), 124-129. Recuperado de http://www.sap.org.ar/docs/publicaciones/archivosarg/2010/v108n2a06.pdf

Cabra, O. L., Infante, D. C., y Sossa, F. N. (2010). El suicidio y los factores de riesgo asociados en niños y adolescentes. Revista Médica Sanitas, 13(2), 28-35. Recuperado de http://www. unisanitas.edu.co/Revista/18/suicidio.pdf

Cañón, S. C. (2011). Factores de riesgo asociados a conductas suicidas en niños y adolescentes. Archivos de Medicina, 11(1), 62-67.

Carmona, A., Arango, C., Castaño, J., Escobar, J., García, C. Godoy, S., et al. (2010). Ca- 
racterización del intento de suicidio en una población ingresada a un hospital infantil de Manizales (Caldas, Colombia), 2000-2008. Archivos de Medicina, 10(1), 9-18. Recuperado de http://www.redalyc.org/articulo. oa?id=273819493001

Carvajal, G., y Caro, C. (2011). Ideación suicida en la adolescencia: una explicación desde tres de sus variables asociadas en Bogotá, 2009. Colombia Médica, 42(29), 45-56. Recuperado de http://www.bioline.org.br/pdf?rc11039

Casullo, M. M. (2004). Ideaciones y comportamientos suicidas en adolescentes: una urgencia social. Facultad de Psicología - UBA / Secretaría de Investigaciones / XII Anuario de Investigaciones, 173-182. Recuperado de https://www.google.com.co/webhp?sourceid =chromenstant\&ion=1\&espv=2\&ie=UTF\#q=Ide aciones+y+comportamientos+suicidas+en+ad olescentes\%3A+Una+urgencia+social

Caycedo, A., Arenas, M. L., Benítez, M., Cavanzo, P., Leal, G., y Guzmán, Y. R. (2010). Características psicosociales y familiares relacionadas con intento de suicidio en una población adolescente en Bogotá, 2009. Persona y Bioética, 14(2), 105-113. Recuperado de http:// personaybioetica.unisabana.edu.co/index. php/personaybioetica/article/view/1807

Chávez, R., Rivera, L., Llerenas, A., Díaz, E., Allen, B., y Lazcano, E. (2009). Factores del abuso sexual en la niñez y la adolescencia en estudiantes de Morelos, México. Revista Saúde Pública, 43(3), 506-514.

Contreras, J. M., Bott, S., Guedes, A., y Dartnall, E. (2010). Violencia sexual en Latinoamérica y el Caribe: análisis de datos secundarios. Iniciativa de Investigación sobre la Violencia Sexual. Recuperado de http://www.oas.org/ dsp/documentos/Observatorio/violencia_sexual_la_y_caribe_2.pdf

Córdova, M. A., Cubillas, M. J., y Román, R. (2011). ¿Es posible prevenir el suicidio? Evaluación de un programa de prevención en estudiantes de bachillerato. Pensamiento Psicológico, 9(17),
21-32. Recuperado de http://www.redalyc. org/articulo.oa?id=80122596002

Córdova, M., Rosales, J., y García, M. (2012). Ideación suicida y variables asociadas en jóvenes universitarios del estado de Hidalgo, México. Psicogente, 15(28), 287-301.

Corte Constitucional. (2006). Ley 1090. Recuperado de http://www.psicologiaprospectiva. com/introley1090.html.

Cortés, R., Cantón-Cortés, D., y Cantor-Duarte, J. (2011). Consecuencias a largo plazo del abuso sexual infantil: papel de la naturaleza y continuidad del abuso y del ambiente familiar. Behavioral Psychology, 19(10), 41-56. Recuperado de https://dialnet.unirioja.es/servlet/ articulo?codigo $=3607870$

Daniel, W. (2006). Bioestadística. Cuarta edición. Limusa Wiley.

Eisenberg, M. E., Ackard, D. M., \& Resnick, M. D. (2007). Protective factors and suicide risk in adolescents with a history of sexual abuse. The Journal of Pediatrics, 151, 482-487.

González, C., Arana, D., y Jiménez, J. (2008). Problemática suicida en adolescentes y el contexto escolar: vinculación autogestiva con los servicios de salud mental. Salud Mental, 31(1), 23-27. Recuperado de http://www. scielo.org. $\mathrm{mx} /$ scielo. php?pid=S0185-3325200 8000100004 \&script=sci_arttext

Hernández, Fernández y Baptista, (2006). Metodología de la investigación. México: editorial: McGraw-Hill.

Instituto Colombiano de Bienestar Familiar. (2012). Revista SUIN, Sistema Único de información de la niñez. Recuperado de http://www.icbf. gov.co/portal/page/portal/PortallCBF/Bienestar/SNBF1/3\%20Revista\%20SUIN.pdf

Instituto Nacional de Medicina Legal y Ciencias Forenses. (2010). Forensis, datos para la vida. Recuperado de http://www.medicinalegal. gov.co/documents/10180/34438/6+SUICIDIO. pdf/3904b04a-4a47-40f6-8359-9a4d83709125 
Instituto Nacional de Medicina Legal y Ciencias Forenses. (2014). Forensis, datos para la vida. Recuperado de http://www.medicinalegal. gov.co/documents/88730/1656998/Forensis+ Interactivo+2014.24-JULpdf.pdf/9085ad79d2a9-4c0d-a17b-f845ab96534b

Instituto Nacional de Salud. (2016). Protocolo de vigilancia en salud pública, intento de suicidio. Colombia.

Mingote, J., Jiménez, M., Osorio, R., y Palomo, T. (2004). Suicidio: asistencia clínica. Guía práctica de psiquiatría médica. Madrid: Díaz de Santos.

Ministerio de Educación Nacional. (1993). Resolución número 8430. Recuperado de https:// www.minsalud.gov.co/sites/rid/Lists/BibliotecaDigital/RIDE/DE/DIJ/RESOLUCION8430-DE-1993.PDF

Muñoz, J., Pinto, V., Callata, H., Napa, N., y Perales, A. (2005). Ideación suicida y cohesión familiar en estudiantes preuniversitarios entre 15 y 24 años. Revista Peruana de Medicina Experimental y Salud Pública, 23(4), 239-246. Recuperado de http://ateneo.unmsm.edu.pe/ ateneo/handle/123456789/891

Organización Mundial de la Salud. (2001). Informe sobre la salud en el mundo 2001. Salud mental, nuevos conocimientos, nuevas esperanzas. Ginebra, Suiza. Recuperado de http:// www.who.int/whr/2001/es/index.html

Organización Mundial de la Salud. (2014). Prevención del suicidio, un imperativo global. Ginebra, Suiza. Recuperado de http://www. who.int/mental_health/suicideprevention/ exe_summary_spanish.pdf?ua $=1$

Organización Mundial de la Salud. (2012). El suicidio, un problema de salud pública enorme $y$, sin embargo, prevenible. Recuperado de http://www.who.int/entity/mediacentre/ news/releases/2004/pr61/es/

Osman, A., Gutiérrez, P., Kopper, B., Barrios, F., y Chiros, C. (1998). The positive and negative suicide ideation inventory: Development and validation. Psychological reports, 82, 783-793. Recuperado de http://www.ncbi.nlm.nih.gov/ pubmed $/ 9676490$

Osman, A., Gutiérrez, P. M., Jiandani, J., Kopper, B. A., Barrios, F. X., Linden, S. C., \& Truelove, R. S. (2003). A preliminary validation of the Positive and Negative Suicide Ideation (PANSI), inventory with normal adolescent samples. PubMed, 59(4), 493-512. Recuperado de http://www.ncbi.nlm.nih.gov/pubmed/12652640

Paniagua, R., González, C., y Rueda, S. (2014). Orientación al suicidio en adolescentes en una zona de Medellín, Colombia. Revista Facultad Nacional de Salud Pública, 32(3), 314321. Recuperado de http://www.redalyc.org/ pdf/120/12031816008.pdf

Pérez, I., Ibáñez, M., Reyes, J., Atuesta, J., y Suárez, M. (2008). Factores asociados al intento suicida e ideación suicida persistente en un Centro de Atención Primaria, Bogotá, 20042006. Revista de Salud Pública, (3), 374-385. Recuperado de http://www.scielosp.org/pdf/ rsap/v10n3/v10n3a02.pdf

Pérez, M., Viacha, M., Martínez, L., y Salas, I. (2013). El maltrato familiar y su relación con la ideación suicida en adolescentes escolarizados de instituciones públicas y privadas de las ciudades de Tunja, Duitama y Sogamoso. Psicogente, 31(17), 80-92. Recuperado de http://publicaciones.unisimonbolivar.edu. co/rdigital/ojs/index.php/psicogente/article/ view/35

Rodríguez, J., Medina, O., y Cardona, D. (2013). Caracterización del suicidio en el departamento de Risaralda, Colombia, 2005-2010. Revista de la Facultad de Medicina, 61(1), 9-16. Recuperado de file:///D:/Users/mpperez/Downloads/39619-176848-1-PB.pdf

Rodríguez, L. (2011). Hacia una comprensión contemporánea del abuso sexual infantil: un diálogo necesario entre la psicología y el derecho. Cultura, Educación y Sociedad, 2(1), 
149-162. Recuperado de http://es.scribd. com/doc/220807992/comprension-contemporanea-ABUSEX\#scribd

Rueda, G., Rangel, A., Castro, V., y Camacho, P. (2010). Suicidabilidad en adolescentes, una comparación con población adulta. Revista Colombiana de Psiquiatría, 39(4), 683-692. Recuperado de http://www.scielo.org.co/ pdf/rcp/v39n4/v39n4a04.pdf

Siabato, E., y Salamanca, Y. (2015). Factores asociados a ideación suicida en universitarios. Psychologia: avances de la disciplina, 9(1), 7181. Recuperado de http://www.scielo.org.co/ pdf/psych/v9n1/v9n1a06.pdf
Toro, R., Avendaño, B., y Castrillón, D. (2016). Design and psychometric analysis of the hopelessness and suicide ideation inventory “IDIS". International Journal of Psychological Research, 9(1), 52-63. Recuperado de http:// www.scielo.org.co/pdf/ijpr/v9n1/v9n1a06.pdf

Ventura, R., Carvajal, C., Undurraga, S., Vicuña, P., Egaña, J., y Garib. (2010). Vigía de los derechos de la niñez y la adolescencia mexicana, 2006. La adolescencia. México.

Villalobos, F. (2010). Ideación suicida en jóvenes: formulación y validación del modelo integrador explicativo en estudiantes de educación secundaria y superior. (Tesis inédita de doctorado). España: Universidad de Granada, España. 